\title{
On the use of isotopic composition measurements of volatile organic compounds to determine the "photochemical age" of an air mass
}

\author{
J. Rudolph and E. Czuba \\ Centre for Atmospheric Chemistry and Chemistry Department, York University, Toronto, Ontario, Canada
}

\begin{abstract}
It is shown that stable carbon isotope ratio measurements in atmospheric volatile organic compounds can be used to determine the photochemical age of the studied compounds. This "Isotopic Hydrocarbon Clock" has several advantages compared to the conventional "Hydrocarbon Clock". Due to the small Kinetic Isotope Effects for the reaction of hydrocarbons with $\mathrm{OH}$-radicals, the photochemical ages derived from changes in isotopic composition are the correct linearly averaged age of the studied compounds, independent of the mixing of air parcels with different photochemical ages. The uncertainties of photochemical ages derived from isotopic composition measurements of atmospheric nonmethane hydrocarbons depend primarily on the accuracy of stable carbon isotope ratio measurements, the Kinetic Isotope Effect for the reaction of hydrocarbons with $\mathrm{OH}$-radicals and the variability of isotopic composition of hydrocarbon sources. Uncertainty analysis shows that the "Isotopic Hydrocarbon Clock" is not only a theoretical possibility but a viable method to determine the photochemical age of atmospheric hydrocarbons.
\end{abstract}

\section{Introduction}

Measurements of reactive trace gases have been used with varying success to determine the extent of photochemical processing in air masses (Roberts et al., 1984; Rudolph and Johnen, 1990; McKeen et al., 1990 and 1996; McKenna et al., 1995; McKenna, 1997). Hydrocarbons are frequently used for this purpose and the technique often is referred to as " $\mathrm{Hy}$ drocarbon Clock". Very recently a method to determine the 13-C/12-C ratio in volatile organic compounds (VOC) has been developed (Rudolph et al., 1997) and successfully applied to a number of atmospheric measurements (Rudolph et al., 1997; Tsunugai et al., 1999). In this paper the possibility to use changes in the isotopic composition of volatile organic compounds to determine the amount of chemical processing for ambient hydrocarbons will be studied and the advantages and limitations of such an "Isotopic Hydrocarbon Clock" discussed.

\section{The Hydrocarbon Clock and Changes in Stable Isotope Ratios in Atmospheric Hydrocarbons}

In case of uniform age $t$ of an air mass the change of the ratio of the concentrations of two trace gases relative to a ref-

Copyright 2000 by the American Geophysical Union.

Paper number 2000GL011385.

0094-8276/00/2000GL011385\$05.00 erence composition can be described by the following frequently used and well known "Hydrocarbon Clock" equation:

$c_{i} / c_{j}={ }^{0} c_{i} / c_{j} * \exp \left(-t / \tau_{j}\right) / \exp \left(-t / \tau_{j}\right)={ }^{0} c_{i} / c_{j} c^{*} \exp \left(-t / \tau_{i}+t / \tau_{j}\right)(1)$

$c_{i}, c_{j}$ : Concentration of substances $i$ and $j$, respectively in the studied air mass.

${ }^{0} c_{i},{ }^{0} c_{j}$ : In the air mass at time $t=0$.

$\tau_{i}, \tau_{j}$ : Atmospheric residence time of compounds $i$ and $j$, respectively.

It has been pointed out in several publications (cf. Rudolph and Johnen, 1990; McKeen et al., 1990 and 1996) that (1) is of limited use in the case of mixing air masses with different extent of chemical processing. For an air mass that is the result of mixing of different air parcels with different "chemical ages" we can describe the concentration by integrating over all relevant ages of the individual air parcels and normalizing to the total air mass:

$$
c_{i}={ }_{0}{ }^{\infty} w(t) *{ }^{0} c_{1}(t) \exp \left(-t / \tau_{i}\right) d t /{ }_{0}{ }^{\infty} w(t) d t
$$

Here $w(t)$ describes the contribution of air parcels of age $t$ and ${ }^{0} c_{i}(t)$ is the initial concentration of component $i$ at $t=0$.

Now we consider that for $t \gg \tau_{i}$, due to the exponential decrease of concentrations with time $c_{i}$ becomes extremely small. We therefore can define a time limit $t_{1}$ such that in any air parcel with $>t_{1}$ the concentrations of compounds $i$ and $j$ are depleted to an extend that these air parcels do not contribute any relevant amount of $i$ or $j$. Then (2) can be written as:

$$
\begin{gathered}
c_{i}=\left[\begin{array}{l}
d{ }^{t l} w(t) \\
* 0
\end{array}{ }^{0} c_{i}(t) \exp \left(-t / \tau_{1}\right) d t+{ }_{t}\right]{ }^{\infty 0} w(t) \\
\left.{ }^{0} c_{1}(t) \exp \left(-t / \tau_{i}\right) d t\right] / 0 \int^{\infty} w(t) d t
\end{gathered}
$$

For practical purposes $t_{i}$ can be defined such that:

$$
{ }_{t} \int^{\infty} w(t) *{ }^{0} c_{i}(t) \exp \left(-t / \tau_{i}\right) d t<c_{p}
$$

With $c_{p}$ being a contribution to $c_{i}$ that is sufficiently small that it can be neglected for any practical purpose. It is obvious, that the value of $t_{1}$ is somewhat arbitrary, but due to the exponential decrease of $c$ with $t / \tau_{i}$ the condition will always be met for a sufficiently large $t_{1}$ and we obtain:

$$
c_{i}=\int_{0}^{t} w(t) *{ }^{0} c_{i}(t) \exp \left(-t / \tau_{i}\right) d t /{ }_{0}^{\infty} w(t) d t
$$

Thus we can describe the ratio of the concentrations of two compounds $i$ and $j$ by:

$c_{i} / c_{j}={ }_{0}^{t t} w(t) *{ }^{0} c_{i}(t) \exp \left(-t / \tau_{i}\right) d t /{ }_{0}{ }^{t} w(t) *{ }^{0} c_{j}(t) \exp \left(-t / \tau_{j}\right) d t$

The interpretation of any results derived from (6) (or any similar treatment) may be difficult if $\tau_{1} \| \tau_{j}$ or $\tau_{i} \| \tau_{j}$. In this case there may be contributions from air parcels that carry information with respect to only one of the two components. 
The temporal scale and consequently the spatial scale that is probed by components with substantially different atmospheric lifetimes may be different.

This problem is avoided if $\tau_{i}=\tau_{j}+\Delta \tau$ with $\Delta \tau$ \& $\tau_{j}$. For the sake of simplicity in the following equations we will use $\tau$ instead of $\tau_{j}$ :

$$
\begin{gathered}
c_{i} / c_{j}={ }_{0} \int^{t} w(t) *{ }^{0} c_{i}(t) \exp \left(-t /(\tau+\Delta \tau) d t / d_{d} \| w(t) *{ }^{0} c_{j}(t)\right. \\
\exp (-t / \tau)
\end{gathered}
$$

If ${ }^{0} c_{i}(t) /{ }^{0} c_{j}(t)$ is independent of $t$, that is the emission ratio of the two compounds is constant, we can substitute ${ }^{0} \mathrm{c}_{\mathrm{i}} /{ }^{\circ} \mathrm{c}_{\mathrm{j}}=$ ${ }^{0} c_{1}(t) /{ }^{0} c_{j}(t)$. Furthermore $t(\tau+\Delta \tau)$ can be approximated by $t / \tau *(1-\Delta \tau / \tau)$ if $\Delta \tau / \tau \ll 1$ and with $\Delta t=t-t_{a v}$ we obtain:

$$
\begin{aligned}
& c_{i} / c_{j}={ }^{0} c_{i} /{ }^{0} c_{j}{ }^{*}{ }_{0}{ }^{\mathrm{d}} w(t) \exp (-t / \tau) * \exp ((t / \tau) \\
& *(\Delta \tau / \tau)) d t /{ }_{0}{ }^{\prime \prime} w(t) \exp (-t / \tau) d t
\end{aligned}
$$

We expand $\exp ((t \tau) *(\Delta \tau / \tau))$ into a power series at $t=t_{\text {av }}$ :

$$
\begin{array}{r}
\exp \left(\mathrm{t} / \tau^{*} \Delta \tau / \tau\right)=\exp \left(\left(\mathrm{t}_{\mathrm{a}} / \tau\right)^{*}(\Delta \tau / \tau)\right) \\
*\left\{1+\Sigma\left[\left((\Delta \mathrm{t} / \tau)^{*}(\Delta \tau / \tau)^{*} 1 / \mathrm{n} !\right)\right]^{\mathrm{n}}\right\}
\end{array}
$$

and if $\left[(\Delta t / \tau)^{*}(\Delta \tau / \tau)\right]$ « 1 or $\Delta t / \tau$ « $\tau / \Delta \tau,(9)$ rapidly converges with increasing $\mathrm{n}$ and we can ignore higher order terms of $\left[\left(\Delta t / \tau^{*} \Delta \tau / \tau^{*} 1 / n !\right)\right]^{n}$. Furthermore, the first order term $(\Sigma$ $\left.\left[\left(\Delta t / \tau^{*} \Delta \tau / \tau\right)\right]\right)$ is zero if we define $t_{\mathrm{av}}$ as average age of compound $\mathrm{j}\left(\mathrm{t}_{\mathrm{av}}=0_{0}{ }^{\infty} \mathrm{c}_{\mathrm{j}}(\mathrm{t}) \mathrm{t} d \mathrm{dt}_{0}{ }^{\infty} \mathrm{c}_{\mathrm{j}}(\mathrm{t}) \mathrm{dt}\right)$. Thus (7) can be written as:

$$
c_{i} / c_{j}=\left({ }^{0} c_{i} /{ }^{0} c_{j}\right) \exp \left(t_{a v} / \tau * \Delta \tau / \tau\right)
$$

or:

$$
\ln \left(c_{i} / c_{j}\right)=\left(t_{a v} / \tau\right)^{*}(\Delta \tau / \tau)+\ln \left({ }^{0} c_{i} /{ }^{0} c_{j}\right)
$$

Upon rearrangement we obtain a possibility to calculate the amount of chemical processing $\left(\mathrm{t}_{\mathrm{av}} / \tau\right)$ in an air mass relative to a reference (initial) composition:

$$
\mathrm{t}_{\mathrm{av}} / \tau=(\tau / \Delta \tau) *\left[\ln \left(\mathrm{c}_{\mathrm{i}} / \mathrm{c}_{\mathrm{j}}\right)-\ln \left({ }^{0} \mathrm{c}_{\mathrm{i}} /{ }^{0} \mathrm{c}_{\mathrm{j}}\right)\right]
$$

A limit for $t_{1}=\tau * \ln \left({ }^{0} c / c_{p}\right)$ can be derived from $c_{p}<{ }^{0} c *$ $\exp (-t / \tau)$ with ${ }^{0} \mathrm{c}$ being the maximum value for the reference concentration of either component $i$ or $j$, whichever requires the higher $t_{1}$. This limit for $t_{1}$ can also be used as limit for $\Delta t$ and we obtain the following condition:

$$
\tau / \Delta \tau » \ln \left({ }^{0} \mathrm{c} / \mathrm{c}_{\mathrm{p}}\right)
$$

Obviously this limits the usefulness of (11) to determine the extent of photochemical processing from atmospheric observations of VOC concentration ratios. It would be extremely fortuitous if the atmospheric residence times of two compounds differ by only a few percent. However, for changes in isotopic composition we usually observe such small differences. Thus (10) or (11) is useful if we define $c_{j} / c_{j}$ as the stable isotope ratio of an atmospheric constituent (z), e.g. a volatile organic compound.

Stable isotope compositions are usually expressed as relative difference between sample and standard (or reference: ref c). Since isotope effects are very small, the relative difference is traditionally expressed in \%o:

$$
\delta_{z}(\%)=\left(c_{i} / c_{j}{ }^{\text {ref }} c_{i}{ }^{\text {ref }} c_{j_{j}}\right) /\left({ }^{\text {ref }} c_{i} /{ }^{\text {ref }} c_{j}\right)^{*} 1000
$$

Here $c_{1}$ and $c_{j}$ denote the abundance of different isotopes in the same compound $(\mathrm{z})$ instead of concentrations of different compounds. Using the delta notation and approximating ln $(1+\delta / 1000)=\delta / 1000$, which is justified for $\delta$ « 1000 , allows to write (10) as:

$$
\delta_{\mathrm{z}}=1000 *\left(\mathrm{tav}_{\mathrm{av}} / \tau_{\mathrm{z}}\right) *\left(\Delta \tau_{\mathrm{z}} / \tau_{\mathrm{z}}\right)+{ }^{0} \delta_{\mathrm{z}}
$$

or

$$
\left(\mathrm{t}_{\mathrm{av}} / \tau\right)=\left(\tau_{z} / \Delta \tau_{\mathrm{z}}\right)^{*}\left(\delta_{\mathrm{z}}-{ }^{0} \delta_{\mathrm{z}}\right) / 1000
$$

$\delta_{2}$ : Delta value (\%o) of compound $\mathrm{z}$ in studied air mass. ${ }^{0} \delta_{\mathrm{z}}$ : Delta value (\%) of compound $\mathrm{z}$ at time zero (emission).

The dominant atmospheric removal mechanism for most $\mathrm{VOC}$ is the reaction with $\mathrm{OH}$ radicals. In this case the atmospheric residence time of a compound $\mathrm{z}$ can be calculated from its rate constant for reaction with $\mathrm{OH}$-radicals $\left({ }^{\mathrm{OH}_{\mathrm{K}}} \mathrm{k}_{\mathrm{z}}\right)$ and the average $\mathrm{OH}$-radical concentration:

$$
\tau_{\mathbf{z}}=1 /\left({ }^{\mathrm{OH}} \mathbf{k}_{\mathbf{z}} *[\mathrm{OH}]\right)
$$

and for $\Delta \tau_{z} \ll \tau_{z}$ :

$$
\Delta \tau_{z} / \tau_{\mathbf{z}}=\Delta{ }^{\mathrm{OH}_{\mathrm{k}}} /{ }^{\mathrm{OH}} \mathrm{k}_{\mathbf{z}}
$$

with $\Delta^{\mathrm{OH}} \mathrm{k}_{\mathrm{z}}$ being the difference in the $\mathrm{OH}$ reaction rate constants of compound $\mathrm{z}$ for different isotopes. Combining (15) with (14) and defining ${ }^{\mathrm{OH}_{\delta}}=\left(\Delta{ }^{\mathrm{OH}_{\mathbf{z}}} \mathrm{PH}_{\mathrm{k}_{2}}\right)^{*} 1000$ gives:

$$
\delta_{\mathrm{z}}=\left(\mathrm{t}_{\mathrm{av}} *{ }^{\mathrm{OH}_{\mathrm{z}}} \mathrm{k}_{\mathrm{z}}[\mathrm{OH}]\right) *{ }^{\mathrm{OH}} \delta+{ }^{\mathrm{O}} \delta_{\mathrm{z}}
$$

or

$$
\mathrm{t}_{\mathrm{av}} *[\mathrm{OH}]=\left(\delta_{\mathrm{z}}-{ }^{0} \delta_{\mathrm{z}}\right) /\left({ }^{\mathrm{OH}} \delta *{ }^{\mathrm{OH}} \mathrm{k}_{\mathrm{z}}\right)
$$

In order to test the practical applicability and usefulness of (16) we have to consider two types of uncertainties. Firstly, are the used assumptions justified. Secondly, can we determine the parameters and variables in (16) with sufficient accuracy to actually make use of (16) to study atmospheric processes.

\section{Uncertainties}

Very recently a study of the Kinetic Isotope Effect (KIE) for the reaction of several non-methane hydrocarbons

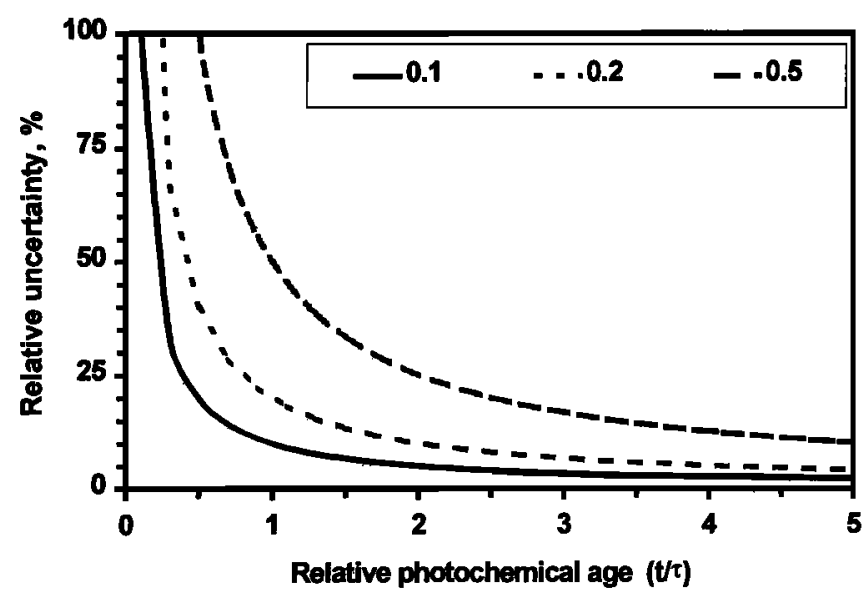

Figure 1. Relative uncertainty of photochemical age as function of ratio of photochemical age over atmospheric lifetime for different values of $\Delta^{0} \delta_{z}{ }^{\mathrm{OH}_{\delta}} \delta$. Details see text. 
(NMHC) with OH-radical was conducted (Czuba, 1999). The KIE ranged from about $1 \%$ to some $10 \%$. As mentioned above, for most VOC reaction with $\mathrm{OH}$ radicals is the dominant atmospheric removal mechanism, and $\tau / \Delta \tau$ will be in the range of $100-1000$. This means that (12) will be valid for ratios of ${ }^{0} \mathrm{c} / \mathrm{c}_{\mathrm{p}}$ up to $10^{40}$, obviously exceeding all imaginable concentration changes in the atmosphere.

From (14a) it follows that the relative uncertainty of photochemical age of a compound is a function of the ratio of age to atmospheric lifetime. Furthermore, the relative uncertainty will depend on the ratio of the uncertainty of ${ }^{0} \delta_{z}$ over the KIE for compound $\mathrm{z}: \Delta^{0} \delta_{z}{ }^{\rho \mathrm{H}} \delta$. Unfortunately, the number of studies of carbon isotope signatures for NMHC emissions into the atmosphere is extremely limited. The only available results are for the greater Toronto area, a region heavily impacted by urban and industrial sources (Czuba, 1999). Although these results are not sufficient to determine typical or representative values for stable carbon isotope composition of NMHC emissions, they indicate that for many compounds the variability is in the range of only a few \%o. Based on this it can be estimated that values for $\Delta^{0} \delta_{z} /{ }^{\mathrm{OH}} \delta$ are between 0.1 to 0.5 . The resulting relative uncertainties of the photochemical age of compound $\mathrm{z}$ are given in Figure 1. For $\Delta^{0} \delta_{z} /{ }^{\mathrm{OH}_{\delta}} \delta$ values below 0.2 reasonable photochemical age estimates can be obtained, except for very low chemical processing. For higher $\Delta^{0} \delta_{z}^{\prime}{ }^{\mathrm{OH}_{3}} \delta$ values the uncertainties increase and reliable calculations of photochemical ages will only be possible for very substantial chemical processing.

The uncertainties of calculations based on (16) also depend on errors of isotopic composition measurements and of the kinetic isotope effects. Based on the few published results of stable carbon isotope ratio measurements in atmospheric VOC, such measurements can be made with uncertainties in the range of 0.3-1 \%o (Rudolph et al., 1997; Tsunugai et al., 1999). The only study of the 13-C/12-C KIE (Czuba, 1999) for the reaction of $\mathrm{NMHC}$ with $\mathrm{OH}$-radicals reports relative uncertainties in the range of $5-20 \%$.

Figure 2 shows resulting uncertainties of photochemical ages. Photochemical ages may be determined with an error of 0.1-0.2 atmospheric lifetimes of the studied compound plus a relative uncertainty in the range of $10-25 \%$ of the "photochemical age". For low photochemical processing the uncertainties are mainly determined by the error of the atmospheric isotope measurements, for "aged" samples the uncertainty of the KIE dominates.

\section{Discussion and Application}

It is important to remember that photochemical ages determined from changes in isotopic composition of atmospheric VOC are a property of the studied compounds, not necessarily of the investigated air mass. The atmospheric lifetime of the studied compound determines the temporal scale, and consequently also the spatial scale that is probed. In this aspect the "isotopic version" of the hydrocarbon clock is similar to the "conventional" approach using concentration ratios instead of stable isotope ratios. However, there are two essential differences. Firstly, the use of isotope ratios gives a linearly weighted average photochemical age of the studied compound, even in the case of mixing of air parcels with nonuniform photochemical ages. Secondly, the use of isotope ratios results in an unambiguous temporal scale. This removes many of the ambiguities that plague the

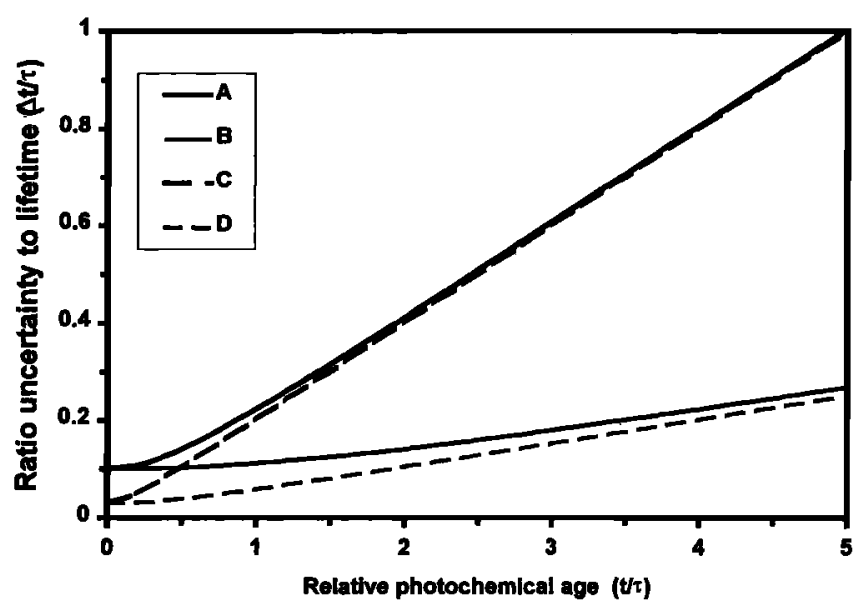

Figure 2. Ratio of uncertainty of photochemical age over atmospheric lifetime as function of the ratio of average photochemical age over lifetime for different errors of stable carbon isotope $\left(\Delta \delta^{13 \mathrm{C}}\right)$ measurements and relative uncertainties of the kinetic isotope effect $(\triangle \mathrm{KIE} / \mathrm{KIE}) . \mathrm{A}$ : $\Delta \mathrm{KIE} / \mathrm{KIE}=0.2, \Delta \delta^{13 \mathrm{C}}=1 \%$; $\mathrm{B}: \Delta \mathrm{KIE} / \mathrm{KIE}=0.05, \Delta \delta^{13 \mathrm{C}}=$ $1 \%$; C: $\Delta \mathrm{KIE} / \mathrm{KIE}=0.2, \Delta \delta^{13 \mathrm{C}}=0.3 \%$; $\mathrm{D}: \Delta \mathrm{KIE} / \mathrm{KIE}=0.05$, $\Delta \delta^{13 \mathrm{C}}=0.3 \%$.

"Conventional Hydrocarbon Clock". One obvious example is that unbiased average $\mathrm{OH}$-radical concentrations can obtained if (16) is combined with an independent estimate of $t_{a v}$, e.g. from meteorology or transport models.

It should be noted that for an isolated air mass $\left(\mathrm{c}={ }^{0} \mathrm{c} *\right.$ $\exp (-t / \tau)),(16)$ can be simplified to a formulation essentially identical to the well known Rayleigh formula:

$$
\delta_{\mathrm{z}}={ }^{\mathrm{OH}} \delta * \ln (\mathrm{c} / \mathrm{c})+{ }^{\circ} \delta_{\mathrm{z}}
$$

Such an idealized approach has been used to derive the KIE for the reaction of ethane with $\mathrm{OH}$-radicals from measurements of the dependence between the $13-\mathrm{C} / 12-\mathrm{C}$ ratio and the concentration of ethane in the atmosphere (Tsunugai et al., 1999). However, the assumption of an isolated air mass is not always justified and thus results derived from atmospheric observations of hydrocarbons using the Rayleigh formulation might be biased and the KIE underestimated.

Based on state of the art techniques, it is to be expected that photochemical ages derived from isotopic composition measurements of atmospheric hydrocarbons will have uncertainties of 0.2-0.5 times the atmospheric residence time of the studied hydrocarbon. Based on average tropospheric $\mathrm{OH}$ radical concentrations of some $10^{6} \mathrm{~cm}^{-3}$ and rate constants for the reaction of reactive hydrocarbons with $\mathrm{OH}$-radicals in the range $10^{-11}$ to $10^{-10} \mathrm{~cm}^{3}$ molecules $\mathrm{s}^{-1} \mathrm{~s}^{-1}$, (cf. Atkinson et al., 1997) isotopic composition measurements might be used to study atmospheric processes on time scales as short as one hour.

As already mentioned, at present there are extremely few measurements of the stable carbon isotope composition of VOC in the atmosphere. The only presently available data set that might be used to calculate "photochemical ages" from isotopic data is a series of measurements in the greater Toronto area in summer 1999 (Czuba, 1999). These measurements include measurements of the $13-\mathrm{C} / 12-\mathrm{C}$ ratio in ambient propene, its KIE for the reaction of propene with 
Table 1. Propene Stable Carbon Isotope Measurements In the Greater Toronto Area

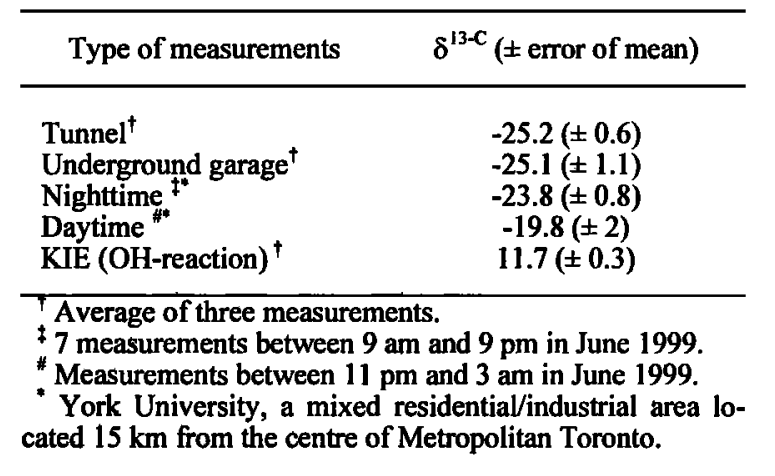

OH-radicals and some source investigations (Table 1). The reaction rate for propene with $\mathrm{OH}$ is relatively high, $2.63 *$ $10^{-11}$ molecules $\mathrm{cm}^{-3} \mathrm{~s}^{-1}$ (Atkinson et al., 1997) and the associated KIE with $11.7 \%$ substantial. Consequently we expect to see some impact of photochemical reaction on isotopic composition even in an urban environment close to emission sources. Indeed, the day/night difference in the isotopic composition is significant, $(4 \pm 2.2 \%)$. Furthermore, the daytime variability of the $\delta^{13 \mathrm{C}}$ is more than twice that at night, compatible with the concept of a significant removal and isotopic fractionation during daytime. The nighttime $\delta^{13 \mathrm{C}}$ of propene is close to that of the sources. The difference of $1.3 \pm$ $1 \%_{0}$ is, due to the small number of source studies, not significant.

Propene also reacts with ozone, however the loss rate due to this reaction during daytime in summer is only a fraction of the $\mathrm{OH}$-radical induced removal. Thus we have good reason to believe that the difference between daytime and nighttime $\delta^{13 \mathrm{C}}$ is due to kinetic isotope fractionation effects associated with the removal of propene by reaction with $\mathrm{OH}$-radicals. In this case we can calculate the extent of photochemical processing of propene. Using (16b) an integrated $\mathrm{OH}$-radical concentration of $(15 \pm 8) * 10^{9} \mathrm{~s}$ molecules $\mathrm{cm}^{-3}$ is obtained, a value that is very well compatible with a summer daytime $\mathrm{OH}$ radical concentrations in the range of several $10^{6} \mathrm{~cm}^{-3}$ and a suburban observation site.

\section{Conclusions}

Measurements of the stable carbon isotope ratio of atmospheric VOC allow the determination of the average photochemical age of the studied compound. For all practical purposes the use of changes in isotopic composition will give the correct, linearly averaged photochemical age, independent of the differences in chemical processing between individual air parcels that contribute to the composition of the investigated air mass. Consequently such studies will be extremely useful to understand photochemistry that is related to the removal of trace gases, as well as the formation of secondary pollutants occurring as consequence of such removal processes. The atmospheric lifetime of the studied component will determine the temporal scale, and thus the spatial scale, of the processes studied. On one hand this may somewhat limit the area that can be probed. On the other hand the possibility to study isotopic composition of compounds with different atmospheric lifetimes provides opportunities to determine the spatial and temporal scales that dominate specific processes, especially since the numerous VOC have widely varying atmospheric lifetimes.

The decrease of concentration with time due to both reaction and dilution will limit the upper end of time scales that can be studied using stable isotope ratios of VOC. In remote areas concentrations of most VOC are well below $1 \mathrm{ppb}$. Nevertheless, the few published studies of stable carbon isotope composition measurements of VOC in remote and semi remote areas (Rudolph et al. 1997; Tsunugai et al.1999) demonstrate that such measurements are possible.

The available data on isotopic composition of sources, kinetic isotope fractionation effects, and atmospheric observations is at present extremely limited. However, the few available data already demonstrate that, using state of the art techniques, isotopic composition measurements of atmospheric VOC will be extremely useful to study photochemical processes in the atmosphere.

Acknowledgement. This research was supported financially by the Natural Science and Engineering Research Council of Canada.

\section{References}

Atkinson, R, D. L. Baulch, R. A. Cox, R. F. Hampson, J. A. Kerr, M. J. Rossi, and J. Troe, Evaluated kinetic, photochemical and heterogeneous data for atmospheric chemistry, supplement V, IUPAC subcommittee on gas kinetic data evaluation for atmospheric chemistry, J. Phys. Chem. Ref. Data, 26, 521 - 1011, 1997.

Czuba, E., Development of a technique to study stable carbon isotope composition of NMHCs in ambient air, Master Thesis, York University, Toronto, Canada, 1999.

McKeen, S. A., M. Trainer, E.-Y. Hsie, R. K. Tallamraju, and S. C. Liu, On the indirect determination of atmospheric OH-radical concentrations from reactive hydrocarbon measurements, J. Geophys. Res., 95, 7493-7500, 1990.

McKeen, S. A., S.C. Liu, E.-Y. Hsie, X. Lin, J. D. Bradshaw, S. Smyth, G. L. Gregory, and D. R. Blake, Hydrocarbon ratios during PEM-WEST A: A model perspective, J. Geophys. Res. 101, 2087-2109, 1996.

McKenna, D. S., C. J. Hord, and J. M. Kent, Hydroxyl radical concentrations and Kuwait oil fire emission rates for March 1991, J. Geophys. Res., 100, 26,005-26,026, 1995.

McKenna, D. S., Analytic solution of reaction diffusion equations and implications for the concept of an air parcel, J. Geophys. Res., $102,19,719-13,725,1997$.

Roberts, J. M., F. C. Fehsenfeld, S. C. Liu, M. J. Bollinger, C. Hahn, D. L. Albritton, and R. E. Sievers, Measurements of aromatic hydrocarbon ratios and $\mathrm{NO}_{\mathrm{x}}$ concentrations in the rural troposphere: Observation of air mass photochemical aging and $\mathrm{NO}_{\mathbf{x}}$ removal, Atmos. Environ., 18, 2421 - 2432, 1984.

Rudolph, J., and F. J. Johnen, Measurements of light atmospheric hydrocarbons over the Atlantic in regions of low biological activity, J. Geophys. Res., 95, 20,583-20,591, 1990.

Rudolph, J., D. C. Lowe, R. J. Martin, and T. S. Clarkson, A novel method for compound specific determination of $\delta 13 \mathrm{C}$ in volatile organic compounds at ppy levels in ambient air, Geophys. Res. Lett., 24, 659-662, 1997.

Tsunugai, U., N. Yoshida, and T. Gamo, Carbon isotopic composition of C2-C5 hydrocarbons and methyl chloride in urban, coastal, and maritime atmospheres over the western North Pacific, J. Geophys. Res., 104, 16,033 - 16,039, 1999.

Czuba, E., J. Rudolph, York University, Centre for Atmospheric Chemistry and Chemistry Department, Toronto, Ontario, M3J 1P3, Canada. (email: rudolphj@yorku.ca)

(Received: January 14, 2000; revised: September 21, 2000; accepted: September 27,2000 ) 